ARTICLE

\title{
Plasmodium sporozoite phospholipid scramblase interacts with mammalian carbamoyl-phosphate synthetase 1 to infect hepatocytes
}

\author{
Sung-Jae Cha ${ }^{1 \times}$, Min-Sik Kim (ib) ${ }^{2}$, Chan Hyun $\mathrm{Na}^{3} \&$ Marcelo Jacobs-Lorena (i) ${ }^{1 凶}$
}

\begin{abstract}
After inoculation by the bite of an infected mosquito, Plasmodium sporozoites enter the blood stream and infect the liver, where each infected cell produces thousands of merozoites. These in turn, infect red blood cells and cause malaria symptoms. To initiate a productive infection, sporozoites must exit the circulation by traversing the blood lining of the liver vessels after which they infect hepatocytes with unique specificity. We screened a phage display library for peptides that structurally mimic (mimotope) a sporozoite ligand for hepatocyte recognition. We identified HP1 (hepatocyte-binding peptide 1) that mimics a $\sim 50 \mathrm{kDa}$ sporozoite ligand (identified as phospholipid scramblase). Further, we show that HP1 interacts with a $160 \mathrm{kDa}$ hepatocyte membrane putative receptor (identified as carbamoylphosphate synthetase 1). Importantly, immunization of mice with the HP1 peptide partially protects them from infection by the rodent parasite $P$. berghei. Moreover, an antibody to the HP1 mimotope inhibits human parasite $P$. falciparum infection of human hepatocytes in culture. The sporozoite ligand for hepatocyte invasion is a potential novel pre-erythrocytic vaccine candidate.
\end{abstract}

\footnotetext{
${ }^{1}$ Johns Hopkins Bloomberg School of Public Health, Department of Molecular Microbiology and Immunology and Malaria Research Institute, 615N. Wolfe St., Baltimore, MD 21205, USA. ${ }^{2}$ Department of New Biology, Daegu Gyeongbuk Institute of Science and Technology (DGIST), Daegu 42988, Republic of Korea.

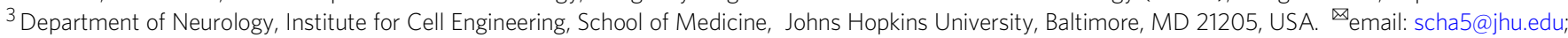
ljacob13@jhu.edu
} 
$\mathrm{M}$ alaria infection is initiated by the release from an infected Anopheles mosquito of 50-100 Plasmodium sporozoites into the skin of a vertebrate host ${ }^{1}$. Thereafter sporozoites migrate through dermal tissues in search of a blood vessel, which they traverse to enter the circulation. Circulating sporozoites must exit in the liver, where they infect hepatocytes, each of which produces thousands of merozoites that are released into the blood circulation and cause disease symptoms ${ }^{2}$. This complex cycle requires specific parasite-host recognition at each stage. Binding of the sporozoite surface circumsporozoite protein (CSP) to liver-specific and highly sulfated glycosaminoglycans (GAGs) protruding into the sinusoidal vessels is the first step for liver recognition and invasion ${ }^{3,4}$. Next, sporozoites glide along the vessel wall and preferentially traverse Kupffer cells via recognition of its CD68 surface receptor ${ }^{5-8}$. After crossing the sinusoid lining, sporozoites specifically invade hepatocytes, no other cell types such as Stellate cells or adipocytes. Thus, we hypothesize that infection requires recognition via interaction of a sporozoite ligand with a hepatocyte receptor. No sporozoite ligand for hepatocyte recognition has been reported. On the other hand, CD81, the scavenger receptor BI (SR-BI), and EphA2 have been proposed as possible hepatocyte receptors for sporozoite invasion ${ }^{9-11}$. The 6-cysteine domain Plasmodium protein P36 was proposed to be involved in the SR-BI-dependent pathway ${ }^{12}$. Subsequently, it was determined that CD81 and SR-BI are not sporozoite receptors but rather, are involved in parasitophorous vacuole membrane formation and organization ${ }^{10}$. Furthermore, a recent report shows that EphA2 is not an obligatory receptor for sporozoite hepatocyte interaction ${ }^{13}$.
Hepatocyte Aquaporin-9 was recently identified as an important host cell membrane protein for sporozoite permissiveness, however was not characterized as a receptor ${ }^{14}$. Hence, the molecular basis for specific sporozoite-hepatocyte interaction remains unknown. The most advanced available RTS/S malaria pre-erythrocytic vaccine that uses CSP as an antigen only shows moderate protection $(\sim 40 \%)^{15}$. Identification of a sporozoite ligand for hepatocyte invasion may identify new pre-erythrocytic malaria vaccine targets.

Here we show that a peptide-HP1-identified by the use of a phage display library, specifically binds to hepatocytes and by doing so, inhibits Plasmodium sporozoite infection. Further, HP1 is a structural mimic of the sporozoite ligand phospholipid scramblase (PLS) that for infection, interacts with the hepatocyte receptor carbamoyl-phosphate synthetase 1 (CPS1).

\section{Results}

The hepatocyte-binding peptide HP1 inhibits sporozoitehepatocyte interaction. We used a two-step strategy to investigate the molecular basis for sporozoite-hepatocyte interaction: (1) use a phage peptide display library to select peptides that strongly bind to the hepatocyte surface and (2) determine whether such peptide competitively inhibits sporozoite-hepatocyte interaction. Should competitive inhibition by a small peptide be observed, this would constitute preliminary evidence that the selected peptide binds to a hepatocyte surface molecule that serves as a sporozoite receptor. We screened a phage library displaying $1.5 \times 10^{9}$ different peptides ${ }^{16}$ for peptides with high affinity to primary mouse hepatocytes (Fig. 1a). Of a $1.5 \times 10^{9}$ peptide displaying phage library
$-3 \times$ repeats $\leftarrow$ Wash \& recover bound phages
Enrichment strong binders

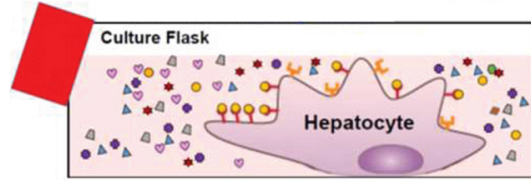

b

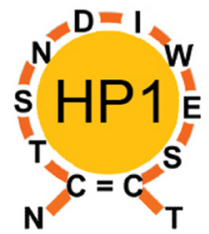

C

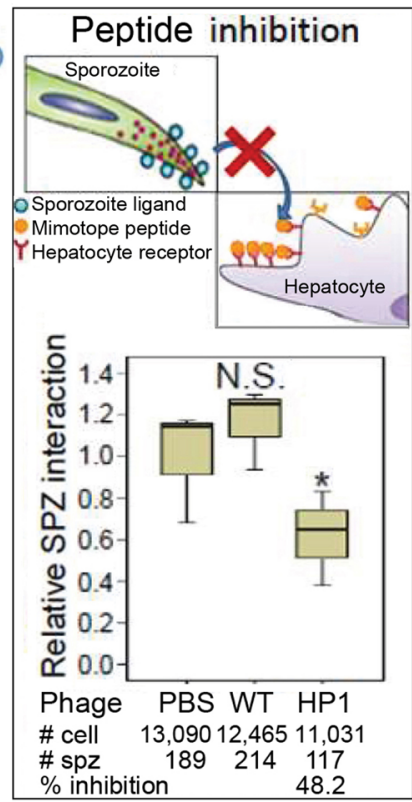

d

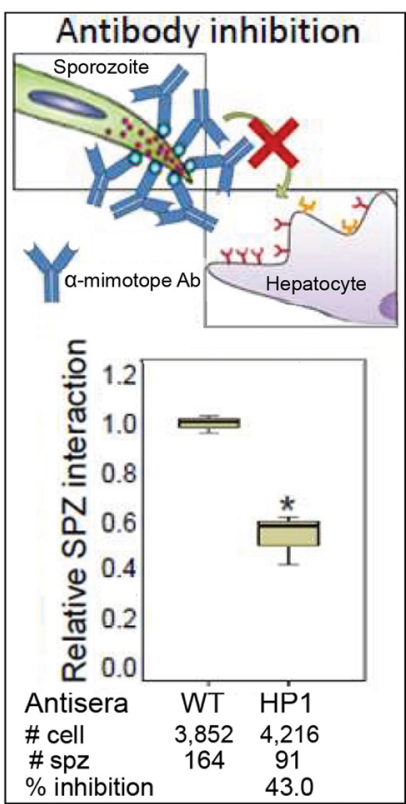

Fig. 1 The HP1 peptide mimics a sporozoite ligand. a Schematic diagram of the screen for peptides that have a strong affinity to primary mouse hepatocyte surface molecules. The phage display library has a complexity of $1.5 \times 10^{9}$ different peptides. $\mathbf{b}$ Amino acid sequence and the basic structure of the strongest hepatocyte binder, HP1 peptide. All library peptides have cysteine at positions 2 and 11 that form a disulfide bond and give the peptide conformation. $\mathbf{c}$ Peptide that mimics the ligand conformation (mimotope) should bind to the hepatocyte receptor and by doing so, inhibit sporozoite-hepatocyte interactions (diagram in the upper panel). Primary mouse hepatocytes were incubated with an HP1 phage or a wild-type phage (non-recombinant; control) and then incubated with $P$. berghei sporozoites. PBS buffer-treated group (no phage) served as another control. Relative sporozoite-hepatocyte interaction was determined by counting the number of sporozoite-bound and -invaded cells/total hepatocyte number (lower panel). Data pooled from three biological replicates. ${ }^{\star} P=0.05$. NS is not significant. $\mathbf{d}$ An antibody that recognizes the mimotope should bind to the sporozoite ligand and by doing so, inhibit sporozoite-hepatocyte interactions (diagram in upper panel). P. berghei sporozoites were pre-incubated for 10 min with sera from mice that were immunized with HP1 phages and then incubated with primary mouse hepatocytes. Relative sporozoite interaction was determined as described above using WT phage antiserum treatment as a control (lower panel). All box plots show medians, quartiles, minimum, and maximums within the bounds of 1.5 times the interquartile range. \% inhibition was calculated using medians. Relative sporozoite interaction was determined by normalizing sporozoite counts compared to the median of the control group, PBS treated, and WT phage antisera treated. Data pooled from three biological replicates. \# cell: total hepatocyte number counted; \# spz: total sporozoite number counted. ${ }^{\star} P=0.05$. $\mathbf{c}$ and $\mathbf{d}$ Two tail $P$-value by Mann-Whitney $U$ test. Source data are provided as a Source Data file. 
39 successfully sequenced phages, 17 (43.6\%) displayed conserved amino acid sequences. Out of these, five displayed an identical peptide that was named HP1 (Fig. 1b, Supplementary Fig. 1). The recombinant HP1 phage, and the wild-type phage that has no peptide insert as control, were tested for competitive inhibition of sporozoite-hepatocyte interaction. The HP1 phage inhibited sporozoite-hepatocyte interaction by $48 \%$ relative to the wild-type phage (Fig. 1c). Additional inhibition assays with sera from mice immunized with the HP1 recombinant phage, or the WT phage as control, revealed that the HP1 antiserum inhibits sporozoite-hepatocyte interactions by $43 \%$ (Fig. 1d).

Further experiments were performed with synthetic peptides, instead of recombinant phages, to control for the possibility of steric hindrance by the $\sim 1 \mu \mathrm{m}$-long phage particles. The HP1 peptide selectively bound to hepatocytes, but not to macrophages, (Fig. 2a) and inhibited sporozoite-hepatocyte interactions by $\sim 51 \%$ relative to the PBS-treated group (Fig. 2b), confirming results of Fig. 1c. The HP1 peptide did not inhibit sporozoite-macrophage interaction. In turn, the unrelated control P39 peptide selectively bound to macrophages, as expected $^{8}$, but not to hepatocytes, and inhibited sporozoite-macrophage interaction, also as expected. Next, mice were immunized with KLH-conjugated HP1 peptide or with non-conjugated KLH as a control. Anti-HP1 sera detected sporozoite surface molecules on unpermeabilized $P$. berghei and P. falciparum sporozoites (Fig. 3a). Immunization with the HP1 peptide rendered mice protected from infection with $48 \%$ efficacy when challenged with the bite of two infected mosquitos (Fig. 3b, left panel). Immunization with HP1 peptide delayed the pre-patent period compared to the control group, which implies sporozoite liver burden was reduced by anti-HP1 antibodies (Supplementary Fig. 2). Additionally, when human parasite $P$. falciparum sporozoites were incubated with antiHP1 sera, interaction with HepG2 human hepatoma cells was inhibited in a dose-dependent manner by up to $87 \%$ (Fig. 3 b, right panel).

Identification of a sporozoite ligand for hepatocyte interaction. The following experiments were performed to test the hypothesis that the HP1 peptide is a mimotope (shares conformation) of a sporozoite surface protein that functions in the interaction with liver cells. This hypothesis predicts that an anti-HP1 antibody
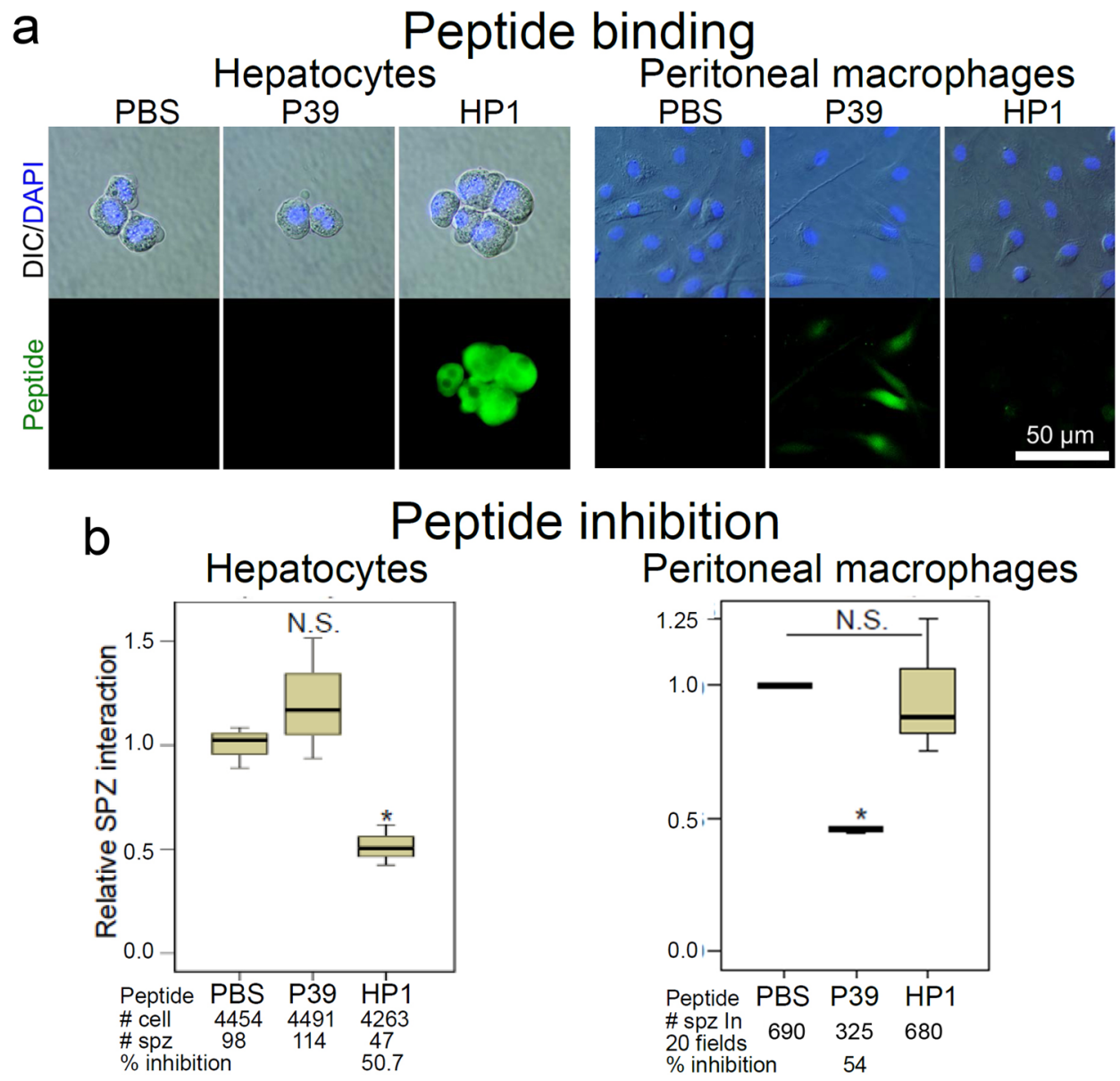

Fig. 2 The synthetic HP1 peptide specifically targets Plasmodium sporozoite-hepatocyte interaction. a A biotin-tagged HP1 peptide was tested for binding to the surface of primary mouse hepatocytes or primary mouse peritoneal macrophages. Peptide binding was detected with Alexa488-conjugated streptavidin. The P39 peptide, which binds to the macrophage surface marker CD688, was used as a negative control for hepatocytes and positive control for macrophages. DIC differential interference contrast microscopy, DAPI: nuclear stain (blue). b The HP1 peptide inhibits sporozoite-hepatocyte interaction by $51 \%$ relative to the PBS-treated control, while it does not inhibit sporozoite-macrophage interaction ( ${ }^{\star} P=0.05$; NS not significant). The P39 peptide inhibits sporozoite-macrophage interaction but does not inhibit sporozoite-hepatocyte interaction ( ${ }^{\star} P=0.04 ;$ NS not significant). Data pooled from three biological repeats. \# cell: total hepatocyte number counted; \# spz: total sporozoite number counted. All box plots show quartiles, medians, minimum, and maximums within the bounds of 1.5 times the interquartile range. The \% inhibition was calculated using medians. Relative sporozoite interaction was determined by normalizing sporozoite counts comparing it to the mean of the PBS-treated control group. Two tail $P$-value by Mann-Whitney $U$ test. ${ }^{\star} P<0.05$. NS is not significant. Source data are provided as a Source Data file. 
also recognizes a sporozoite ligand (see Fig. 1d diagram). Western blotting of $P$. berghei sporozoite lysates with an anti-HP1 antibody detected a $\sim 50 \mathrm{kDa}$ protein, a putative sporozoite ligand (Fig. 4a). Although this $\sim 50 \mathrm{kDa}$ band is at a similar position as a cleaved CSP band, the anti-HP1 antibody did not detect a band at the position of the larger uncleaved CSP protein, indicating that the HP1 peptide is not a CSP mimotope. This was confirmed by determining that the anti-HP1 antibody does not recognize an immunoprecipitated CSP protein (Supplementary Fig. 3). Additional Western blotting assays show that the expression of the $\sim 50 \mathrm{kDa}$ putative sporozoite ligand is limited to mature salivary

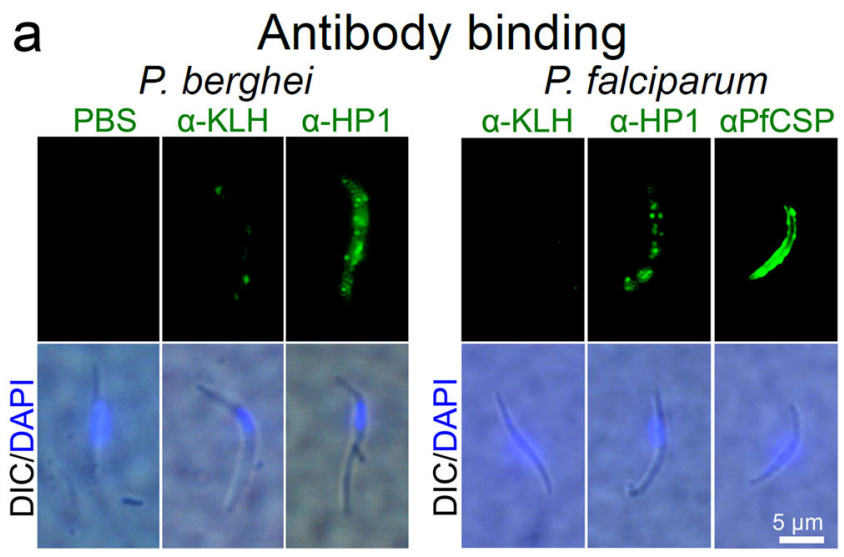

\section{b Antibody inhibition}

Immunization \& In vitro antibody inhibition of $\mathrm{Pb}$ SPZ challenge Pf SPZ-HepG2 cell interaction
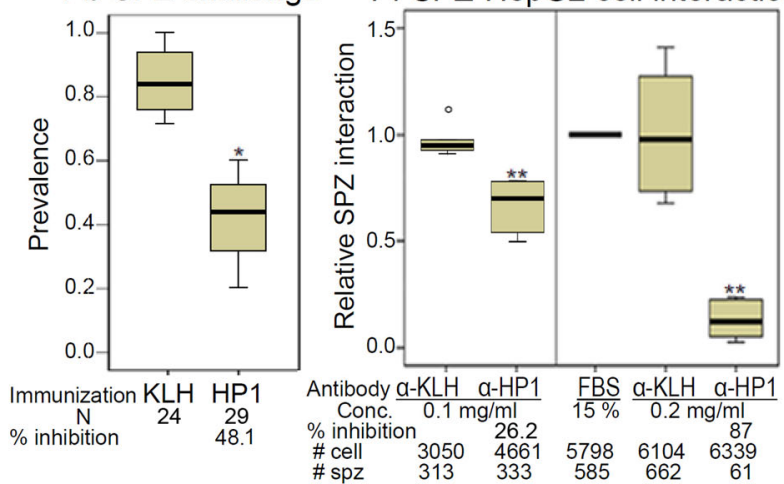

Fig. 3 An anti-HP1 peptide antibody binds to the Plasmodium sporozoite surface and inhibits sporozoite-hepatocyte interaction. a Binding of an anti-HP1 peptide antibody to non-permeabilized P. berghei and P. falciparum sporozoites was detected with Alexa488-conjugated secondary antibody. b left panel, Mice were immunized with KLH-conjugated HP1 peptide, and 10 days after the third boost, the mice were challenged by the bite of two infected mosquitoes. Immunization with KLH alone served as a control. The prevalence of mouse infection was determined by Giemsa staining of thin blood smears ( $\left.{ }^{\star} P=0.02\right)$. Data pooled from four biological repeats. $N$ : number of mice challenged. $\mathbf{b}$ right panel, $P$. falciparum sporozoites were pre-incubated for $10 \mathrm{~min}$ with sera from mice that were immunized with $\mathrm{KLH}$-conjugated HP1 or with non-conjugated $\mathrm{KLH}$, and then incubated with HepG2 human hepatoma cells for $1 \mathrm{~h} .{ }^{\star \star} P=0.002$ in the left and $P=0.004$ in the right. Data pooled from two biological repeats. All box plots show quartiles, medians, minimum, and maximums within the bounds of 1.5 times the interquartile range. A dot represents an outlier beyond the upper bound. The \% inhibition was calculated using medians. Relative sporozoite interaction was determined by normalizing sporozoite counts compared to the mean of the anti-KLH antibody-treated control group. Two tail $P$-value by Mann-Whitney $U$ test. Source data are provided as a Source Data file. gland sporozoites (Fig. 4b). Mass spectroscopic analysis of the $\sim 50 \mathrm{kDa}$ sporozoite proteins from a gel piece shown in Fig. $4 \mathrm{a}$ (stained gel, red box) identified seven sporozoite-specific proteins,
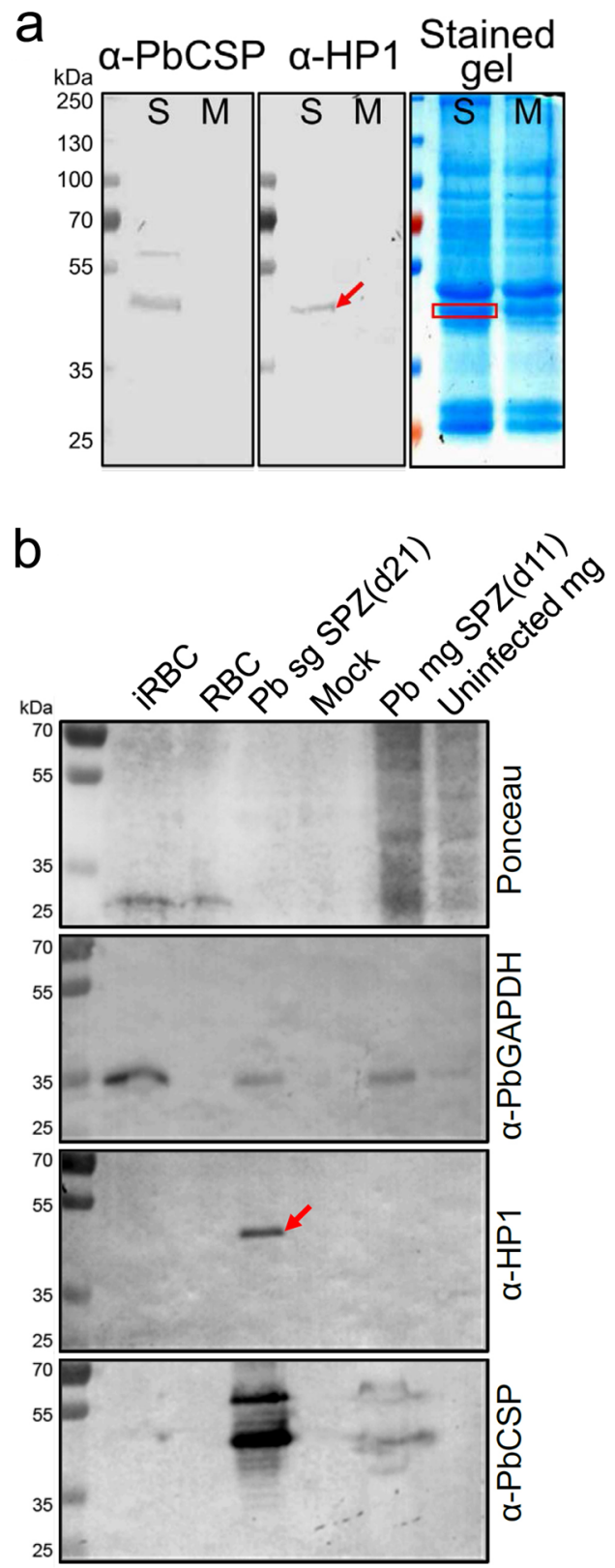

Fig. 4 An anti-HP1 antibody recognizes a $\sim \mathbf{5 0}$ kDa sporozoite ligand protein. a An anti-HP1 antibody recognizes a $\sim 50 \mathrm{kDa}$ protein band (red arrow) from sporozoites isolated from infected mosquito salivary glands $(S)$ but not from mock preparations from uninfected mosquito salivary glands $(M)$. A red box on the stained gel denotes the gel piece that was cut for mass spectroscopy analysis. b Anti-HP1 antibody recognizes a protein specific to mature sporozoites. Lysates of asexual blood-stage parasites (iRBC), purified salivary gland sporozoites (Pb sg SPZ) 21 days after infection, and immature midgut sporozoites ( $\mathrm{Pb} \mathrm{mg} \mathrm{SPZ}$ ) 11 days after infection were fractionated by SDS-PAGE. Uninfected RBCs, sporozoite preparations from uninfected mosquito salivary glands (Mock), and uninfected midguts were used as controls. Ponceau stain served as a loading reference and an anti-PbGAPDH antibody served as a positive control for $P$. berghei parasite lysates. The anti-HP1 antibody detected a $\sim 50 \mathrm{kDa}$ protein only from salivary gland sporozoites (red arrow). $\mathbf{a}$ and $\mathbf{b}$ Each image represents two independent repeats. Source data are provided as a Source Data file. 
including CSP (Table 1). PlasmoDB (plasmodb.org) predicts that the six proteins and CSP are expressed on the sporozoite surface. These proteins were expressed using a bacterial expression system (Fig. 5a). Expression was confirmed using an anti-His tag antibody (red arrows in the second panel) and anti-CSP antibody (green arrow in the third panel). Importantly, the anti-HP1 antibody recognized only one of the proteins (red arrow in the bottom panel), PLS. Specific recognition of the recombinant PbPLS by an anti-HP1 antibody was confirmed with ELISA assays (Supplementary Fig. 4). The PLS role as a sporozoite ligand for hepatocyte interaction is supported by the observation that recombinant PLS binds to the surface of hepatocytes (Fig. 5b). To test whether an anti-PLS antibody inhibits sporozoite-hepatocyte interaction, mice were immunized with the recombinant PbPLS protein or with the empty pET tag protein as a control. AntiPbPLS antibodies recognized the HP1 peptide (Supplementary Fig. 5) and Western blots from sporozoite lysates probed with the anti-PbPLS antibody and the anti-HP1 antibody detected proteins with the same electrophoretic mobility (Supplementary Fig. 6). Importantly, anti-PbPLS antibodies bound to the surface of unpermeabilized P. berghei sporozoites (Fig. 6a), and by doing so inhibited sporozoite-primary mouse hepatocyte interaction by 78\% (Fig. 6b). Additionally, anti-PbPLS antibodies cross-reacted with $P$. falciparum sporozoite surface PLS (Fig. 6c) and similarly inhibited sporozoite-Huh7 human hepatoma cell interaction in a dose-dependent manner (Fig. 6d).

Identification of a hepatocyte receptor for sporozoite interaction. To identify the HP1-binding target on the hepatocyte surface, a recombinant tetrameric HP1 peptide was produced using a bacterial expression system (Supplementary Fig. 7a). We could not use a biotin-tagged HP1 peptide because hepatocytes have many endogenously biotinylated proteins. The recombinant pETHP1 peptide bound to mouse hepatocytes (Supplementary Fig. 7b) and inhibited P. falciparum sporozoite-HepG2 human hepatoma cell interaction in a dose-dependent manner (Supplementary Fig. 7c). Hepatocyte membrane, cytosolic, and insoluble fractions were fractionated by SDS-PAGE followed by farWestern blotting with the recombinant pET-HP1 peptide, or with empty pET tag protein as a control. This assay identified a $\sim 160 \mathrm{kDa}$ hepatocyte membrane protein as a target of the recombinant pET-HP1 peptide binding (Fig. 7a). As an alternative approach, pull-down of primary mouse hepatocyte membrane proteins with the recombinant pET-HP1 peptide identified a protein of the same size (Fig. 7b, red arrow). Mass spectroscopy analysis of the $\sim 160 \mathrm{kDa}$ proteins from the gel in Fig. $7 \mathrm{~b}$ (red arrow) identified five candidate hepatocyte membrane proteins (Table 2). Of these, only CPS1 has been shown to localize to the cell surface ${ }^{17}$. We confirmed that CPS1, an abundant liver protein, is exposed on the surface of primary mouse hepatocytes (Fig. 8a). Although another candidate VWA8 (von Willebrand factor type A domain-containing protein; Table 2) has a wellknown extracellular protein domain (vWA) ${ }^{18}$, its presence on the hepatocyte surface could not be detected (Fig. 8a). In a complementary approach, we pulled down hepatocyte proteins with the recombinant sporozoite PLS protein. Western blotting of the eluates determined that PLS pulls down hepatocyte CPS1 (Fig. 8b). Further, direct interaction between the recombinant PbPLS and hepatocyte CPS1 proteins was verified with ELISA assays (Fig. 8c).

\section{Discussion}

Previously, our group pioneered the use of phage peptide display library screening to investigate the molecular basis of Plasmodium

Table 1 Mass spectroscopy analysis of a gel piece (red box in Fig. 4a) identified seven sporozoite surface proteins.

\begin{tabular}{lllll} 
PlasmoDB ID & \# peptide & Spz surface reported & GPI anchor & Master protein descriptions \\
\hline PBANKA_0510200 & 1 & Yes & No & Conserved Plasmodium protein, unknown function \\
PBANKA_1422900 & 2 & Yes & No & Conserved Plasmodium protein, unknown function \\
PBANKA_1034500 & 2 & Yes & No & Conserved Plasmodium protein, unknown function \\
PBANKA_1240600 & 2 & Yes & No & Inner membrane complex protein 1g, putative \\
PBANKA_1026200 & 13 & Yes & No & NADP-specific glutamate dehydrogenase, putative \\
PBANKA_0506900 & 1 & Yes & No & Phospholipid scramblase, putative \\
PBANKA_0403200 & 1 & Yes & Yes & CSP \\
\hline
\end{tabular}
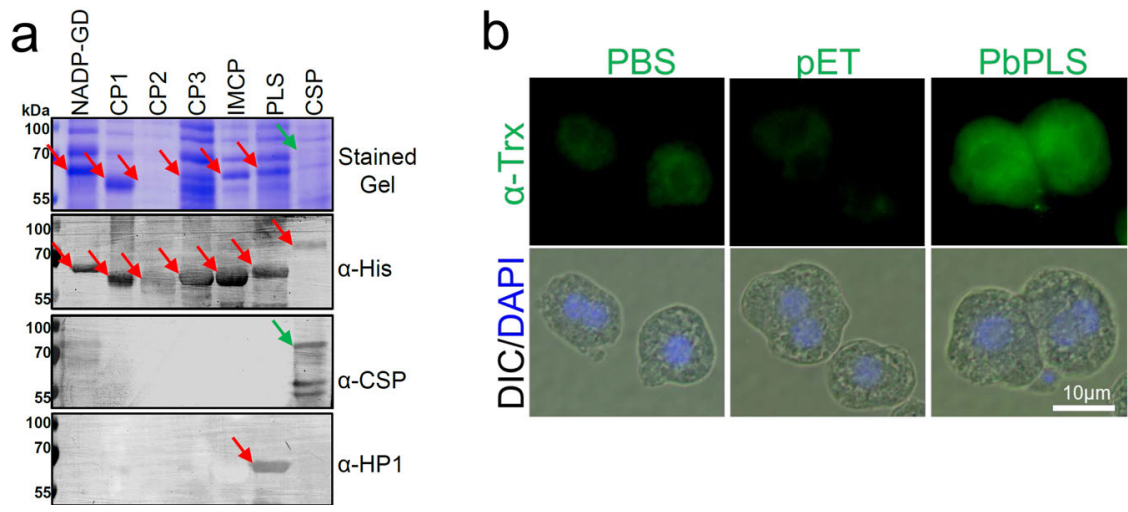

Fig. 5 The anti-HP1 antibody recognizes sporozoite scramblase. a The seven genes listed in Table 1 were expressed as recombinant proteins and fractionated by SDS-PAGE. Western blotting with an anti-His tag antibody confirmed expression of the recombinant proteins (red arrows) and an anti-CSP antibody confirmed CSP expression (green arrow). Importantly, the anti-HP1 antibody only recognized the scramblase (PLS) protein (red arrow). b the PLS protein binds to hepatocytes. Recombinant PLS, pET tag protein, or PBS were incubated with primary mouse hepatocytes. Protein binding was visualized with an anti-thioredoxin-tag antibody. $\mathbf{a}$ and $\mathbf{b}$ Each image represents two independent repeats. DIC differential interference contrast microscopy; DAPI nuclear stain (blue). Source data are provided as a Source Data file. 

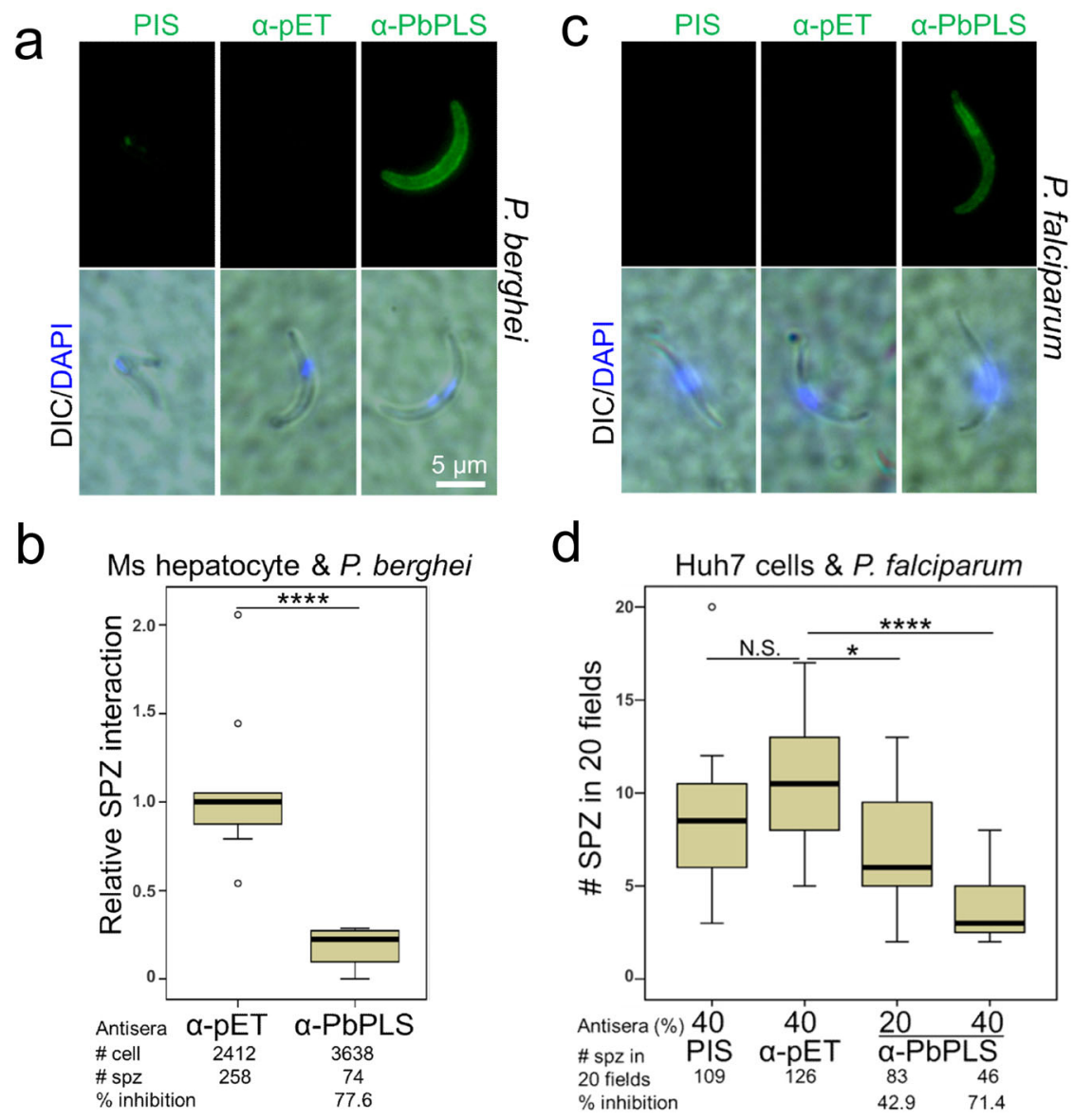

Fig. 6 The anti-PbPLS antibody binds to the sporozoite surface and inhibits sporozoite-hepatocyte interaction. a Immunofluorescent assays show that an anti-PbPLS antibody binds to unpermeabilized P. berghei sporozoites. Antibody binding was visualized with Alexa488-conjugated secondary antibody. Preimmune sera (PIS) or anti-pET tag antibody do not bind. b An anti-PbPLS serum strongly inhibits $P$. berghei sporozoite-primary mouse (Ms) hepatocyte interaction ( $\left.{ }^{\star \star \star \star} P<0.0001\right)$. \# cell: total hepatocyte number counted; \# spz: total sporozoite number counted. c An anti-PbPLS antibody binds to unpermeabilized $P$. falciparum sporozoites. d An anti-PbPLS antibody inhibits $P$. falciparum sporozoite-human hepatoma Huh7 cell interaction in a dosedependent manner ( ${ }^{\star} P=0.02 ;{ }^{\star \star \star \star} P<0.0001$; NS not significant). All box plots show quartiles, medians, and maximum within the bounds of 1.5 times interquartile range. Dots represent outliers beyond the upper and lower bounds. The \% inhibition was calculated using medians. Relative sporozoite interaction was determined by normalizing sporozoite counts comparing to the mean of the anti-pET antibody-treated control group. Data pooled from two independent assays. Two tail $P$-value by Mann-Whitney $U$ test. $\mathbf{a}$ and $\mathbf{c}$ Each image represents two independent repeats. Source data are provided as a Source Data file.

ookinete-mosquito midgut interaction, Plasmodium fertilization, sporozoite-mosquito salivary gland interaction, and sporozoi te-Kupffer cell interaction $8,17,19-23$. An important feature of this approach is that it does not require a priori knowledge of any property of the interacting partner proteins. In this study, we performed two independent screens of a phage library to select peptides that bind to primary mouse hepatocytes. Both screens selected the same HP1 peptide with an overall $44 \%$ frequency, indicating that biopannings reliably lead to enrichment of the strongest binder.

Our attempt to immunoprecipitate the $\sim 50 \mathrm{kDa}$ sporozoite protein recognized by an anti-HP1 antibody was not successful, perhaps because of poor solubility of the PLS membrane lipid raft protein that scrambles membrane phospholipids ${ }^{24,25}$. Recently the scramblase activity of $P$. falciparum PLS was confirmed, and deletion of the gene did not affect blood-stage parasite growth ${ }^{26}$. PLS, whose presence on the surface of the salivary gland-sporozoites had been reported ${ }^{27}$, may not use enzyme activity for interaction with its liver receptor because the enzyme-substrate pocket resides within the parasite plasma membrane, not in the extracellular domain ${ }^{28}$. Other functional roles attributed to PLS are the interaction of this surface protein with extracellular signaling molecules ${ }^{29}$ and mediation of hepatitis $\mathrm{C}$ virus entry into the host cell by interacting with viral envelop proteins ${ }^{30}$. As is the case for PLS and CPS1, our previous studies found that enolase on the surface of ookinetes and GAPDH on the surface of sporozoites also perform moonlighting functions as parasite ligands ${ }^{19,23}$.

PLS is well conserved among Plasmodium species $(\sim 75 \%$ identity) and poorly conserved with mammalian proteins $(\sim 21 \%$ identity between P. falciparum and human PLS). Furthermore, PfPLS has low polymorphism: only two non-synonymous SNPs were identified, with $1 \%$ frequency each out of 219 sequenced strains (plasmodb.org). This low polymorphism may be due to the short exposure time of this sporozoite-stage specific protein to the host immune system. Moreover, low polymorphism bodes well for the potential use of PLS as an additional vaccine antigen. Plasmodium CSP, the most abundant sporozoite surface protein and antigen of present vaccine trials, has 46 non-synonymous SNPs out of 213 sequenced strains, some alleles occurring with $46 \%$ frequency (plasmodb.org). Although the latest R21 malaria vaccine on trial, which uses the central repeat and C-terminus of the PfCSP, reported $\sim 75 \%$ of protective efficacy ${ }^{31}$, large-scale field trial may encounter reduction of protective efficacy due to the 

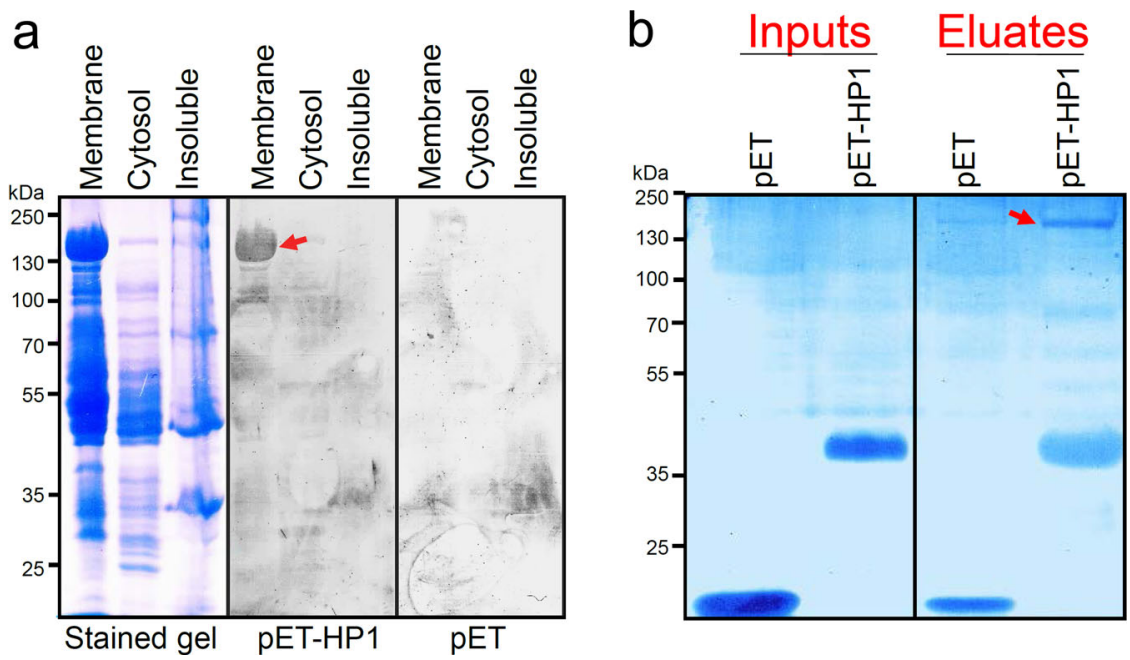

Fig. 7 The HP1 peptide and recombinant PLS bind to a $\mathbf{- 1 6 0} \mathbf{~ k D a}$ hepatocyte membrane protein. a Far-Western blotting assay of mouse primary hepatocyte fractions using recombinant pET-HP1 peptide or $19 \mathrm{kDa}$ empty $\mathrm{pET}$ tag protein as a control. Recombinant pET-HP1 binding to a $160 \mathrm{kDa}$ hepatocyte membrane protein (red arrow) was detected with an anti-tag antibody. b Primary mouse hepatocyte membrane proteins were incubated either with empty recombinant pET tag or recombinant pET-HP1 peptide. The pET-HP1 peptide specifically pulled down a 160 kDa mouse hepatocyte membrane protein (red arrow). Coomassie blue-stained gel. $\mathbf{a}$ and $\mathbf{b}$ Each image represents two independent repeats. Source data are provided as a Source Data file.

Table 2 Mass spectroscopy analysis of a gel band (red arrow in Fig. 7b) identified five hepatocyte putative membrane proteins.

\begin{tabular}{lllll} 
Uniprot ID & \# peptide & Cell surface reported & Transmembrane domain & Description \\
\hline Q8C196 & 279 & Yes & Yes & Carbamoyl-phosphate synthase, Cps1 \\
Q8CC88 & 111 & No & Yes & von Willebrand factor A domain-containing protein 8, Vwa8 \\
Q9DBM2 & 81 & No & Yes & Peroxisomal bifunctional enzyme, Ehhadh \\
Q6PB66 & 105 & No & Yes & Leucine-rich PPR motif-containing protein, Lrpprc \\
F8WHL2 & 43 & No & Yes & Coatomer subunit alpha, Copa \\
\hline
\end{tabular}

polymorphisms in the C-terminal region, as was the case for the RTS,S malaria vaccine ${ }^{32}$.

CPS1 is a putative hepatocyte receptor because it physically interacts with the sporozoite ligand PLS and because binding of the HP1 mimotope to CPS1 inhibits sporozoite-hepatocyte interaction. CPS1 is reported to be a mitochondrial enzyme involved in the urea cycle. However, it is also constitutively secreted and aggregated on the hepatocyte surface ${ }^{33}$. Functional role of the hepatocyte CPS1 as a sporozoite receptor has never been explored and appears to have this moonlighting function. CPS1 secretion increases upon liver injury and circulating CPS1 binds to macrophages, acting as a protective cytokine that inhibits inflammation ${ }^{33}$. Prior to the final productive infection, Plasmodium sporozoites migrate through several hepatocytes by breaching their plasma membrane ${ }^{34}$. This suggests that hepatocyte injury activates CPS1 secretion and inhibits liver macrophage inflammation, in this way supporting early liver stage parasite growth.

In summary, we identified a hepatocyte-binding peptide-HP1, that interacts with the CPS1 hepatocyte surface protein (putative receptor) and whose conformation mimics the sporozoite PLS surface protein (putative ligand). Hepatocyte CPS1 directly interacts with recombinant sporozoite PLS. Importantly, interference with this interaction represses sporozoite infectivity and immunization with HP1 partially protects mice from Plasmodium infection. Targeting sporozoite PLS-hepatocyte CPS1 interaction should be explored as a novel malaria control strategy.

\section{Methods}

Animal ethics protocol. This study was performed in strict accordance with the recommendations from the Guide for Care and Use of Laboratory Animals of the National Institutes of Health (NIH). The animal use was done in accordance with protocol MO21H18 approved by the Johns Hopkins University Animal Care and Use Committee

Cell culture and sporozoite isolation. For isolation of mouse hepatocytes, a $\sim 20 \mathrm{~g}$ male or female C57BL/6J mouse was perfused through the portal vein with $0.05 \%$ collagenase type IV for $15 \mathrm{~min}$ at $37^{\circ} \mathrm{C}$. After perfusion, liver cells were dissociated, and non-parenchymal cells were removed with 3 -min centrifugation at $50 \times g$, repeated three times. The hepatocyte-containing pellet was resuspended in DMEM medium and dead cells were removed by $25 \%$ Percoll centrifugation at $500 \times g$ for $10 \mathrm{~min}$. Live hepatocytes in $25 \%$ Percoll were transferred to fresh DMEM medium supplemented with $10 \%$ heat-inactivated fetal bovine serum (hiFBS). Primary mouse peritoneal macrophages were isolated from a C57BL/6J mouse as previously described $^{22}$ and incubated in the same medium. Briefly, $4 \mathrm{ml}$ of PBS was injected into the peritoneum and then carefully retrieved using a $20 \mathrm{G}$ needle. After centrifugation at $500 \times g$ for $4 \mathrm{~min}$ in a $15 \mathrm{ml}$ tube, the cell pellet was resuspended in $2 \mathrm{ml}$ Ack lysis buffer (Gibco) to remove RBC. After centrifugation at $500 \times g$ for 4 min, peritoneal cells were resuspended in DMEM medium with $10 \%$ hiFBS and cultured in eight-well chamber slides (Thermo Scientific). All procedures were executed in accordance with a protocol approved by the Johns Hopkins University Animal Care and Use Committee. Mouse rooms are maintained at 30-70\% relative humidity and a temperature of $18-26^{\circ} \mathrm{C}\left(64-79^{\circ} \mathrm{F}\right)$ providing $14 \mathrm{~h}$ light/10 h dark Human hepatoma cells, HepG2 and Huh7, were maintained in the DMEM medium with $10 \%$ hiFBS. P. falciparum (NF54) or P. berghei (tdTomato-expressing ANKA 2.34$)^{35}$ sporozoites were isolated from infected female An. stephensi mosquitoes by dissecting salivary glands ${ }^{22}$.

Peptide screening using a phage display library. Isolated $10^{7}$ primary hepatocytes were incubated with $2 \times 10^{11}$ library phages ${ }^{15}$ for $30 \mathrm{~min}$ in a $1.5 \mathrm{ml}$ tube with intermittent agitation. Hepatocytes were then transferred to a $15 \mathrm{ml}$ tube and washed six times with $3 \mathrm{ml}$ PBS. After the final wash, hepatocytes were resuspended in $3 \mathrm{ml}$ of host $E$. coli ( $\sim 10^{8}$ cells) culture in LB broth and incubated for $10 \mathrm{~min}$ at $37^{\circ} \mathrm{C}$ to allow bound phages to infect host $E$. coli. Phages were amplified by culturing the infected bacteria overnight in $100 \mathrm{ml} \mathrm{LB}$ broth with tetracycline ${ }^{15}$ Amplified phages were precipitated by adding to the culture supernatant, the same volume of 16\% PEG8000 (Fisher Scientific) in PBS. After three additional rounds of selection using fresh primary mouse hepatocytes, the selected bound phages were 


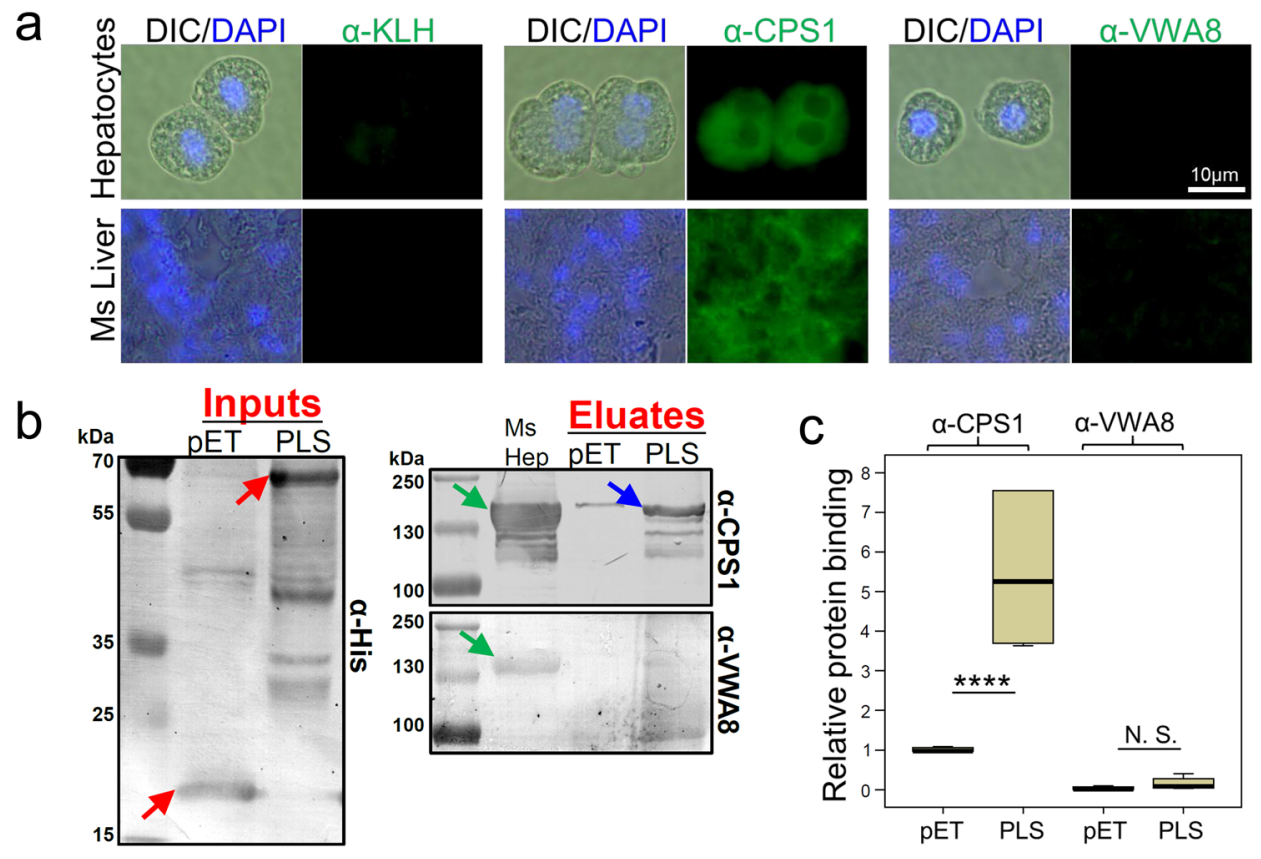

Fig. 8 Sporozoite PLS binds to hepatocyte CPS1. a Immunofluorescence assays show that CPS1 is present on the surface of non-permeabilized primary mouse hepatocytes. DIC differential interference contrast microscopy, DAPI nuclear stain (blue). Ms liver mouse liver sections. b Tagged recombinant sporozoite PLS and pET tag proteins (red arrows) were attached to S-tag agarose beads and incubated with lysates of primary mouse hepatocytes (Ms Hep). Bound hepatocyte proteins were eluted into loading buffer and fractionated by SDS-PAGE. Western blotting (antibody indicated to the right of the panels) shows that liver CPS1 binds to the recombinant sporozoite PLS protein (blue arrow). Hepatocyte lysate was used as a positive control to verify antiCPS1 and anti-vWA8 antibody binding (green arrows). c An ELISA assay shows that the recombinant sporozoite PbPLS interacts with the recombinant mouse hepatocyte CPS1. Ni-coated ELISA plate was incubated overnight with recombinant sporozoite PbPLS or pET tag protein as a control. After blocking, mouse hepatocyte lysate was added to each well. Hepatocyte CPS1 or VWA8 protein binding was determined using anti-CPS1 or anti-VWA8 antibodies, alkaline phosphatase-conjugated secondary antibody, and colorimetric substrate. The protein-binding intensity was normalized to anti-S-tag antibody binding, which determines the quantity of the recombinant protein bound to the plate ( ${ }^{\star \star \star \star} P<0.0001$; NS not significant). Data pooled from two independent experiments. All box plots show quartiles, medians, and maximum within the bounds of 1.5 times interquartile range. Two tail $P$-value was calculated with the one-way ANOVA TukeyHSD test. $\mathbf{a}$ and $\mathbf{b}$ Each image represents two independent repeats. Source data are provided as a Source Data file.

recovered, amplified in E. coli, and plated on a tetracycline LB agar plate. The peptide-coding DNA from 48 randomly picked phage colonies from two independent experiments was PCR amplified. Of these, 39 were successfully sequenced ${ }^{15}$.

Recombinant protein expression. $P$. berghei sporozoite candidate proteins (Fig. 5a), pET-HP1 peptide (Fig. 7), and $\sim 19 \mathrm{kDa}$ empty pET tag protein (thioredoxin-tag, $\mathrm{N}$ - and $\mathrm{C}$-terminal His-tags, and S-tag) were expressed using the pET32b expression system (Novagen) and BL21(DE3) E. coli. All DNA inserts for recombinant protein expression were synthesized by BioBasic Inc (www.biobasic.com). Protein expression was induced by the addition of $0.4 \mathrm{mM}$ IPTG for $4 \mathrm{~h}$. For purification of recombinant proteins, the induced cell pellet was lysed in $1 \%$ Triton X100. Cell lysates were incubated with S-tag agarose (Millipore) for the purification of recombinant proteins. Bound protein was eluted with low $\mathrm{pH}$ buffer and elution buffer was exchanged with PBS by dialysis.

Immunofluorescence assays. Mammalian cells or sporozoites were fixed in 4\% paraformaldehyde in PBS for $30 \mathrm{~min}$ and blocked with 4\% BSA in PBS for $1 \mathrm{~h}$. Mouse frozen liver sections were fixed in methanol and blocked with $4 \%$ BSA in PBS. Sporozoite-infected mammalian cells were permeabilized in 0.2\% Triton X100 in PBS for $15 \mathrm{~min}$ after fixation. Immunofluorescence assays for sporozoite surface molecules used $0.5 \%$ antisera in PBS. Peptide or recombinant protein-binding assays used $200 \mu \mathrm{g} / \mathrm{ml}$ of peptide or recombinant protein. We used commercially available anti-S-tag (Novagen), anti-His tag (Genescript), anti-CPS1(Proteintech), anti-vWA8 (Invitrogen), and Alexa488-conjugated secondary (Invitrogen) antibodies under the manufacturer's directions.

In vitro sporozoite inhibition assays. For in vitro sporozoite inhibition assays, sporozoites were incubated with host cells (one sporozoite per two host cells) for $3 \mathrm{~h}$ allowing initial binding and invasion, and sporozoite attachment and invasion was measured. $P$. berghei experiments used freshly isolated $2 \times 10^{5}$ primary mouse hepatocytes in $1.5 \mathrm{ml}$ tube and $P$. falciparum experiments used two human hepatoma cell lines-HepG2 and Huh7-in eight-well chamber slide (Labtek-II),
$10^{5}$ host cells per chamber were added for monolayer culture, to confirm that the results are not cell-specific. For inhibition assays, cells were incubated with $10^{10}$ phages $/ \mathrm{ml}, 400 \mu \mathrm{g} / \mathrm{ml}$ peptide, $0.1-0.2 \mathrm{mg} / \mathrm{ml}$ purified antibody or $20-50 \%$ antisera in culture medium. Sporozoite-host cell interaction was determined by counting bound plus invaded sporozoites after triple washes with PBS in 20 microscopic fields under $\times 400$ magnification with triplicated tubes, $\sim 5 \mu \mathrm{l}$ of cell suspension containing $\sim 10^{3}$ mouse hepatocytes (Figs. 1, 2a, and 6b), or chambers, human hepatoma cell monolayer (Figs. 2b, 3b right panel, 6d, Supplementary Fig. 7c), per treatment which covers $\sim 40 \%$ assayed cells. Bound and invaded sporozoites were identified with an anti-CSP antibody and Alexa488-conjugated secondary antibody after permeabilization. Relative sporozoite interaction was determined by normalizing sporozoite counts comparing to the mean of control group.

Immunization and antibodies. For immunization and challenge assays, $21-25 \mathrm{~g}$ female Swiss Webster mice were injected with $50 \mu \mathrm{g}$ per mouse of each KLHconjugated peptide or with KLH alone using 50\% AddaVax adjuvant (InvivoGen). Priming subcutaneous injection was followed by triple boosts at two-week intervals. At 10 days after the last boost, immunized mice were challenged with P. berghei sporozoites via biting by two infected An. stephensi mosquitoes. For mouse challenge assays, highly infected female mosquitoes were selected by examination of salivary gland fluorescence, 18 days after mosquito feeding on mice infected with tdTomato-expressing parasites ${ }^{24}$. Mouse parasite infection was determined by thin blood smears followed by Giemsa staining from days 4 to 12 post-challenge. Polyclonal anti-PbPLS or anti-pET tag antisera were generated by immunization of mice with $50 \mu \mathrm{g}$ of recombinant protein per mouse with $50 \%$ AddaVax adjuvant (InvivoGen).

Western and far-Western blotting assays. Parasites, host cells, and recombinant proteins were harvested, pelleted, resuspended with $1 \times$ SDS gel loading buffer, and boiled for $10 \mathrm{~min}$. Protein lysates were fractionated by $10 \%$ SDS-PAGE and then stained with Coomassie dye or transferred onto a PVDF membrane. Membranes were incubated for $1 \mathrm{~h}$ in blocking buffer $(0.1 \%$ Tween 20 and $4 \%$ milk powder in PBS), followed by the addition of a primary antibody. 
Antibody binding was visualized with alkaline phosphatase-conjugated secondary antibody (Promega). For the far-Western blotting assay in Fig. $7 \mathrm{a}$, mouse hepatocytes were used for sub-cellular fractionation as previously described $^{8}$. A total of $1-2 \times 10^{7}$ primary mouse hepatocytes were harvested with buffer 1 (0.01\% digitonin, $10 \mathrm{mM}$ PIPES, $\mathrm{pH} 6.8,300 \mathrm{mM}$ sucrose, $100 \mathrm{mM}$ $\mathrm{NaCl}, 3 \mathrm{mM} \mathrm{MgCl}$, and $5 \mathrm{mM} \mathrm{EDTA}$ ) and rotated for $10 \mathrm{~min}$ at $4{ }^{\circ} \mathrm{C}$. After 1 -min centrifugation $(16,900 \times g)$, the supernatant (cytosolic fraction) was collected, and the cell pellet was washed with buffer 1 . The pellet was then resuspended with buffer $2(0.5 \%$ Triton X-100, $10 \mathrm{mM}$ PIPES, pH 7.4, $300 \mathrm{mM}$ sucrose, $100 \mathrm{mM} \mathrm{NaCl}, 3 \mathrm{mM} \mathrm{MgCl}_{2}$, and $3 \mathrm{mM} \mathrm{EDTA}$ ) and rotated for $20 \mathrm{~min}$ at $4{ }^{\circ} \mathrm{C}$. After 1 -min centrifugation $(16,900 \times g)$, the supernatant (membrane fraction) was collected, and the cell pellet was washed with buffer 2 . The pellet was then resuspended in $6.5 \mathrm{M}$ urea (insoluble fraction). Each fraction was fractionated by $10 \%$ SDS-PAGE and transferred onto a PVDF membrane. The membrane was incubated in $0.1 \mathrm{mg} / \mathrm{ml}$ of recombinant pET tag or pET-HP1 peptide. Protein binding was detected with an anti-S-tag antibody.

ELISA. Recombinant protein or biotinylated peptide was attached to a $\mathrm{Ni}$ coated or a streptavidin-coated ELISA plate (Thermo Fisher), respectively. After washing and blocking with $1 \%$ BSA in PBS, the coated wells were further incubated with antibody or hepatocyte lysate. An anti-S tag antibody determined the amount of recombinant protein bound to the Ni-coated well for normalization. Anti-HP1 antibody binding was quantified by alkaline phosphatase-conjugated secondary antibody (Supplementary Figs. 4 and 5) and binding hepatocyte proteins CPS1 or VWA8 to PLS was quantified by using a non-tagged primary antibody and alkaline phosphatase-conjugated secondary antibodies (Fig. 8c).

Pull-down assays. For pull-down assays (Figs. 7b and 8b), recombinant pET-HP1, pET-PLS, or pET tag protein was bound to S-tag agarose beads (Millipore) and incubated overnight with hepatocyte lysate in $1 \%$ Triton X100. After washing, bound proteins were eluted by boiling S-tag agarose beads in $1 \times$ SDS gel loading buffer. Western blotting assays with an anti-CPS1 or anti-VWA8 antibody visualized bound protein to the beads.

Statistical analyses. For ELISA assays we used the one-way ANOVA Tukey honest significant differences (Tukey HSD) test for two tail $P$-value using SPSS (12.0) software. For Kaplan-Meier test we used GraphPad Prism (9.2.0) for a two-tail $P$-value. For infection and inhibition assays we used Mann-Whitney $U$ test for the two-tail $P$-value. The number of independent repeats is specified in the legends.

Reporting summary. Further information on research design is available in the Nature Research Reporting Summary linked to this article.

\section{Data availability}

All experimental data were listed accordingly. Source data are provided with this paper as Source Data files. Detailed protein information in Tables 1 and 2 can be found in https:// plasmodb.org/plasmo/app/record/gene/PBANKA_0510200, https://plasmodb.org/ plasmo/app/record/gene/PBANKA_1422900, https://plasmodb.org/plasmo/app/record/ gene/PBANKA_1034500, https://plasmodb.org/plasmo/app/record/gene/ PBANKA_1240600, https://plasmodb.org/plasmo/app/record/gene/PBANKA_1026200, https://plasmodb.org/plasmo/app/record/gene/PBANKA_0506900, https:// plasmodb.org/plasmo/app/record/gene/PBANKA_0403200, and https:// www.uniprot.org/uniprot/Q8C196, https://www.uniprot.org/uniprot/Q8CC88, https:// www.uniprot.org/uniprot/Q9DBM2, https://www.uniprot.org/uniprot/Q6PB66, https:// www.uniprot.org/uniprot/F8WHL2. Source data are provided with this paper.

Received: 6 June 2021; Accepted: 4 November 2021; Published online: 19 November 2021

\section{References}

1. Kebaier, C., Voza, T. \& Vanderberg, J. Kinetics of mosquito-injected Plasmodium sporozoites in mice: fewer sporozoites are injected into sporozoite-immunized mice. PLoS Pathog. 5, el000399 (2009).

2. Sturm, A. et al. Manipulation of host hepatocytes by the malaria parasite for delivery into liver sinusoids. Science 313, 1287-1290 (2006).

3. Frevert, U. et al. Malaria circumsporozoite protein binds to heparan sulfate proteoglycans associated with the surface membrane of hepatocytes. J. Exp. Med. 177, 1287-1298 (1993).

4. Pradel, G., Garapaty, S. \& Frevert, U. Proteoglycans mediate malaria sporozoite targeting to the liver. Mol. Microbiol. 45, 637-651 (2002).
5. Vreden, S. G. The role of Kupffer cells in the clearance of malaria sporozoites from the circulation. Parasitol. Today 10, 304-308 (1994).

6. Pradel, G. \& Frevert, U. Malaria sporozoites actively enter and pass through rat Kupffer cells prior to hepatocyte invasion. Hepatology 33, 1154-1165 (2001).

7. Frevert, U. et al. Intravital observation of Plasmodium berghei sporozoite infection of the liver. PLoS Biol. 3, e192 (2005).

8. Cha, S. J. et al. CD68 acts as a major gateway for malaria sporozoite liver infection. J. Exp. Med. 212, 1391-1403 (2015)

9. Silvie, O. et al. Hepatocyte CD81 is required for Plasmodium falciparum and Plasmodium yoelii sporozoite infectivity. Nat. Med. 9, $93-96$ (2003).

10. Yalaoui, S. et al. Scavenger receptor BI boosts hepatocyte permissiveness to Plasmodium infection. Cell Host Microbe 4, 283-292 (2008).

11. Kaushansky, A. et al. Malaria parasites target the hepatocyte receptor EphA2 for successful host infection. Science 350, 1089-1092 (2015).

12. Manzoni, G. et al. Plasmodium P36 determines host cell receptor usage during sporozoite invasion. Elife 6, https://doi.org/10.7554/eLife.25903 (2017).

13. Langlois, A. C., Marinach, C., Manzoni, G. \& Silvie, O. Plasmodium sporozoites can invade hepatocytic cells independently of the Ephrin receptor A2. PLoS ONE 13, e0200032 (2018).

14. Amanzougaghene, $\mathrm{N}$. et al. The host protein aquaporin-9 is required for efficient Plasmodium falciparum sporozoite entry into human hepatocytes. Front. Cell. Infect. Microbiol. 11, 704662 (2021).

15. Tran, T. M., Portugal, S., Draper, S. J. \& Crompton, P. D. Malaria vaccines: moving forward after encouraging first steps. Curr. Trop. Med. Rep. 2, 1-3 (2015).

16. Bonnycastle, L. L., Mehroke, J. S., Rashed, M., Gong, X. \& Scott, J. K. Probing the basis of antibody reactivity with a panel of constrained peptide libraries displayed by filamentous phage. J. Mol. Biol. 258, 747-762 (1996).

17. Cha, S. J., McLean, K. J. \& Jacobs-Lorena, M. Identification of Plasmodium GAPDH epitopes for generation of antibodies that inhibit malaria infection. Life Sci. Alliance 1, e201800111 (2018).

18. Colombatti, A. \& Bonaldo, P. The superfamily of proteins with von Willebrand factor type A-like domains: one theme common to components of extracellular matrix, hemostasis, cellular adhesion, and defense mechanisms. Blood 77, 2305-2315 (1991).

19. Ghosh, A. K., Coppens, I., Gardsvoll, H., Ploug, M. \& Jacobs-Lorena, M. Plasmodium ookinetes coopt mammalian plasminogen to invade the mosquito midgut. Proc. Natl Acad. Sci. USA 108, 17153-17158 (2011).

20. Vega-Rodriguez, J., Perez-Barreto, D., Ruiz-Reyes, A. \& Jacobs-Lorena, M. Targeting molecular interactions essential for Plasmodium sexual reproduction. Cell. Microbiol. 17, 1594-1604 (2015).

21. Ghosh, A. K. et al. Malaria parasite invasion of the mosquito salivary gland requires interaction between the Plasmodium TRAP and the Anopheles saglin proteins. PLoS Pathog. 5, e1000265 (2009).

22. Vega-Rodriguez, J. et al. Multiple pathways for Plasmodium ookinete invasion of the mosquito midgut. Proc. Natl Acad. Sci. USA 111, E492-E500 (2014).

23. Cha, S. J., Kim, M. S., Pandey, A. \& Jacobs-Lorena, M. Identification of GAPDH on the surface of Plasmodium sporozoites as a new candidate for targeting malaria liver invasion. J. Exp. Med. 213, 2099-2112 (2016).

24. Merregaert, J. et al. Phospholipid scramblase 1 is secreted by a lipid raftdependent pathway and interacts with the extracellular matrix protein 1 in the dermal epidermal junction zone of human skin. J. Biol. Chem. 285, 37823-37837 (2010)

25. Hankins, H. M., Baldridge, R. D., Xu, P. \& Graham, T. R. Role of flippases, scramblases and transfer proteins in phosphatidylserine subcellular distribution. Traffic 16, 35-47 (2015).

26. Haase, S. et al. Identification and characterisation of a phospholipid scramblase in the malaria parasite Plasmodium falciparum. Mol. Biochem. Parasitol. 243, 111374 (2021).

27. Lindner, S. E. et al. Total and putative surface proteomics of malaria parasite salivary gland sporozoites. Mol. Cell. Proteom. 12, 1127-1143 (2013).

28. Feng, S. et al. Cryo-EM studies of TMEM16F calcium-activated ion channel suggest features important for lipid scrambling. Cell Rep. 28, 1385 (2019).

29. Nanjundan, M. et al. Plasma membrane phospholipid scramblase 1 promotes EGF-dependent activation of c-Src through the epidermal growth factor receptor. J. Biol. Chem. 278, 37413-37418 (2003).

30. Gong, Q. et al. Phospholipid scramblase 1 mediates hepatitis $C$ virus entry into host cells. FEBS Lett. 585, 2647-2652 (2011).

31. Datoo, M. S. et al. Efficacy of a low-dose candidate malaria vaccine, R21 in adjuvant Matrix-M, with seasonal administration to children in Burkina Faso: a randomised controlled trial. Lancet 397, 1809-1818 (2021).

32. Chaudhury, S. et al. Breadth of humoral immune responses to the C-terminus of the circumsporozoite protein is associated with protective efficacy induced by the RTS,S malaria vaccine. Vaccine 39, 968-975 (2021).

33. Park, M. J. et al. Constitutive release of CPS1 in bile and its role as a protective cytokine during acute liver injury. Proc. Natl Acad. Sci. USA 116, 9125-9134 (2019). 
34. Mota, M. M. et al. Migration of Plasmodium sporozoites through cells before infection. Science 291, 141-144 (2001).

35. Graewe, S., Retzlaff, S., Struck, N., Janse, C. J. \& Heussler, V. T. Going live: a comparative analysis of the suitability of the RFP derivatives RedStar, mCherry and tdTomato for intravital and in vitro live imaging of Plasmodium parasites. Biotechnol. J. 4, 895-902 (2009).

\section{Acknowledgements}

This work was supported by grant AI080668 from the National Institute of Allergy and Infectious Diseases (NIAID). We thank the Johns Hopkins Malaria Research Institute mosquito and parasite core facilities for help with mosquito rearing and $P$. falciparum gametocyte production. Support from the Johns Hopkins Malaria Research Institute and the Bloomberg Philanthropies is gratefully acknowledged. The supply of human blood was supported by the National Institutes of Health Grant RR00052.

\section{Author contributions}

S.-J.C. ${ }^{*}$ contributed with experimental work and the writing of the manuscript. M.-S.K. and C.H.N. performed mass spectroscopy experiments. M.J.-L. ${ }^{*}$ contributed with the experiment design and writing of the manuscript.

\section{Competing interests}

The authors declare no competing interests.

\section{Additional information}

Supplementary information The online version contains supplementary material available at https://doi.org/10.1038/s41467-021-27109-7.
Correspondence and requests for materials should be addressed to Sung-Jae Cha or Marcelo Jacobs-Lorena.

Peer review information Nature Communications thanks Jetsumon Sattabongkot, Melanie Shears and the other, anonymous, reviewer(s) for their contribution to the peer review of this work.

Reprints and permission information is available at http://www.nature.com/reprints

Publisher's note Springer Nature remains neutral with regard to jurisdictional claims in published maps and institutional affiliations.

(c) (i) Open Access This article is licensed under a Creative Commons Attribution 4.0 International License, which permits use, sharing, adaptation, distribution and reproduction in any medium or format, as long as you give appropriate credit to the original author(s) and the source, provide a link to the Creative Commons license, and indicate if changes were made. The images or other third party material in this article are included in the article's Creative Commons license, unless indicated otherwise in a credit line to the material. If material is not included in the article's Creative Commons license and your intended use is not permitted by statutory regulation or exceeds the permitted use, you will need to obtain permission directly from the copyright holder. To view a copy of this license, visit http://creativecommons.org/ licenses/by/4.0/.

(C) The Author(s) 2021 